\title{
TAME SURFACES AND TAME SUBSETS OF SPHERES IN $E^{3}$
}

\author{
BY \\ L. D. LOVELAND(1)
}

1. Introduction. Let $M$ be a connected 2-manifold in $E^{3}$ and let $V$ be one of the components of $E^{3}-M$. We say that $M$ can be locally spanned in $V$ if and only if for each point $p \in M$ and for each positive number $\varepsilon$ there is an $\varepsilon$-disk $R$ in $M$ such that $p \in \operatorname{Int} R$ and for each positive number $\alpha$ there is an $\varepsilon$-disk $D$ in $V$ and a homeomorphism of $\mathrm{Bd} R$ onto $\mathrm{Bd} D$ which moves points no more than a distance $\alpha$. Burgess raised the following question when he proved that $M$ is tame from $V$ if $M$ can be uniformly locally spanned in $V$ [11, Theorem 13].

QUESTION. Is $M$ tame from $V$ if $M$ can be locally spanned in $V$ ?

We define $M$ to be tame from $V$ if $M+V$ is a 3-manifold with boundary. Although we do not answer the above question, it provided the motivation for most of the results in this paper. An affirmative answer to the question would remove the uniform condition from the hypothesis of Theorem 13 of [11].

If in addition to the conditions of the above definition we also require that the boundary of $R$ be tame, then we answer the above question in the affirmative (Theorem 5). The hypothesis that $\mathrm{Bd} R$ is tame seems less restrictive in view of the fact that for each $p \in M$ there is a nested sequence of disks in $M$ such that $p$ is their intersection and each disk has a tame boundary [6, Theorem 1]. Theorem 5 is a special case of Theorem 4. Theorems 2 and 4 depend on Theorems 1 and 3, respectively. The proofs given for Theorems 1 and 3 are similar, but the proof for Theorem 1 is less complicated. It is for this reason that Theorems 1 and 2 precede Theorems 3,4, and 5. Although we shall state our definitions and theorems for surfaces in $E^{3}$, similar results can be obtained for 2-manifolds in a 3-manifold. To see how this can be done, see $\S 6$ of [11].

Theorems 2, 5, and 7 of this paper and Theorems 8 and 13 of [11] are related to the above question. Using results in [17], we are able to modify the proofs of some of these theorems to give sufficient conditions for certain closed subsets of 2-spheres to lie on tame 2-spheres. An indication of the nature of these modifications is given in $\$ 5$.

Presented to the Society, April 18, 1964 under the title $A$ characterization of tame sufaces in $E^{3}$ and April 24, 1964 under the title Tame subsets of spheres in $E^{3}$; received by the editors May 29, 1965.

(1) This paper contains part of the author's doctoral thesis which was written at the University of Utah under the direction of Professor C. E. Burgess. Much of this work was supported by a National Aeronautics and Space Administration fellowship. 
For each 2-sphere $S$ in $E^{3}$, for each $p \in S$, and for each positive number $\varepsilon$, there is a 2 -sphere $S^{\prime}$ of diameter less than $\varepsilon$ such that $p$ is contained in the interior of $S^{\prime}$. In $\S 6$ we give an additional condition on $S \cap S^{\prime}$ which implies that $S$ is tame. Harrold [14] proved that $S$ is tame provided $S \cap S^{\prime}$ is a simple closed curve with an additional requirement which, as proved earlier by Harrold, Griffith, and Posey [15], is equivalent to the requirement that $S \cap S^{\prime}$ be tame. Burgess $[11$, Theorem 8] later showed that $S$ is tame without requiring that the simple closed curve $S \cap S^{\prime}$ be tame. Theorem 12 shows that $S$ is tame if we impose the additional requirement that $S \cap S^{\prime}$ is a continuum satisfying Property ( $*, S \cap S^{\prime}, S$ ), which is defined below. Theorem 12 does not include the result proved by Burgess because a simple closed curve $J$ must be tame to satisfy Property $(*, J, S)\left[13\right.$, Theorem 10]. In fact $S \cap S^{\prime}$ must be tame (that is, $S \cap S^{\prime}$ must lie on a tame 2-sphere) if Property $\left(*, S \cap S^{\prime}, S\right)$ is satisfied [17, Theorem 6]. However in view of the result by Harrold, Griffith, and Posey [15] mentioned above and in view of Gillman's result [13] that a tame simple closed curve $J$ on $S$ satisfies Property $(*, J, S)$, Theorem 12 does include Harrold's result, and it allows $S \cap S^{\prime}$ to be more general than a tame simple closed curve. For example $S \cap S^{\prime}$ can be a connected tame finite graph, a tame Sierpiński curve, or any connected finite union of sets $G$ which satisfy either of Properties $(*, G, S)$ or $(*, G)$ as defined below. In this connection, see Theorems 16, 17, 19, and 21 of [17].

Most of the definitions and notation will be the same as in [5], [11], and [17]. We say that a simple closed curve $J$ can be shrunk to a point in a set $Y$ if each homeomorphism $f$ of the boundary of a disk $D$ onto $J$ can be extended to map (continuously) all of $D$ into $Y$. A 2-manifold $M$ in $E^{3}$ is tame if and only if there is a homeomorphism $h$ of $E^{3}$ onto itself such that $h(M)$ is a polyhedron. We define a closed subset $G$ of a 2-sphere in $E^{3}$ to be tame if $G$ lies on some tame 2-sphere in $E^{3}$.

Consider a closed subset $G$ of a 2 -sphere $S$ in $E^{3}$. Properties ( $\left.*, G, S\right),(A, G, S)$, $\left(A^{\prime}, G, S\right)$, and $(*, G)$ are defined in $[17]$, but we repeat the definitions here because these properties appear frequently in this paper. We use the prefix in " $\varepsilon$-disk," " $\varepsilon$-subset," etc., to imply that the set in question has diameter less than $\varepsilon$. A simple closed curve is unknotted if it bounds a tame disk in $E^{3}$.

Property $(*, G, S)$. It follows from Bing's Side Approximation Theorem [8, Theorem 16] that for each component $V$ of $E^{3}-S$ and for each positive number $\varepsilon$ there is a polyhedral 2-sphere $S^{\prime}$ containing a finite collection $D_{1}, D_{2}, \cdots, D_{n}$ of disjoint $\varepsilon$-disks, and there is a finite collection $E_{1}, E_{2}, \cdots, E_{r}$ of disjoint $\varepsilon$-disks on $S$ such that

1. there is a homeomorphism of $S$ onto $S^{\prime}$ that moves no point as much as $\varepsilon$,

2. $S^{\prime}-\Sigma D_{i} \subset V$, and

3. $S \cap S^{\prime} \subset \Sigma E_{i}$. 
Property $(*, G, S)$ holds if and only if the Side Approximation Theorem can be applied in such a way that

4. $\left(\Sigma E_{i}\right) \cap G=\varnothing$.

Property $(A, G, S)$. We define Property $(A, G, S)$ to mean that for each positive number $\varepsilon$ there is a positive number $\delta$ such that each $\delta$-simple closed curve in $E^{3}-S$ can be shrunk to a point in an $\varepsilon$-subset of $E^{3}-G$.

Property $\left(A^{\prime}, G, S\right)$. Property $\left(A^{\prime}, G, S\right)$ holds if and only if for each positive number $\varepsilon$ there is a positive number $\delta$ such that each unknotted simple closed curve of diameter less than $\delta$ lying in $E^{3}-S$ is the boundary of a tame $\varepsilon$-disk in $E^{3}-G$.

Property $(*, G)$. The closed set $G$ satisfies Property $(*, G)$ if and only if each 2-sphere which contains $G$ and is locally tame modulo $G$, is tame.

Property $(*, G, S)$ insures that $G$ is tame $[17$, Theorem 6], and each of Properties $(A, G, S)$ and $\left(A^{\prime}, G, S\right)$ is sufficient for $G$ to be tame if the diameters of the components of $G$ have a positive lower bound [17, Theorems 10,13, and 6]. We use these results several times in giving sufficient conditions for closed subsets of spheres to be tame.

In several of the proofs which follow we use the concept of linking, relative to simple closed curves, as defined by Bing in [2]. A proof for Lemma 1 can be obtained using the results and definitions in $\S 9$ of that paper. A proof of Lemma 2 is given in [11, Lemma 1]. When we want to show that Property $(A, G, S)$ holds, we rely on Lemma 3, which is easily established. A proof for Lemma 4, which is a generalization of a theorem proved by Bing [5, Theorem 5], can be found in [11, Theorem 1].

LEMMA 1. If $J$ and $K$ are simple closed curves which link in $E^{3}$, then $J$ cannot be shrunk to a point in the complement of $K$.

LEMMA 2. If $D_{1}, D_{2}, \cdots, D_{n}$ is a finite collection of disjoint disks in $E^{3}$ and $f$ is a map of a disk $K$ into $E^{3}$ such that $f(\mathrm{Bd} K) \subset E^{3}-\Sigma D_{i}$, then there is a map $g$ of $K$ into $E^{3}$ such that

1. $g|\mathrm{Bd} K=f| \mathrm{Bd} K$,

2. $g(K) \subset f(K)+\Sigma$ Int $D_{i}$, and

3. $g(K)-\Sigma D_{i}$ is connected.

LEMMA 3. If $G$ is a closed subset of a 2-sphere $S$ in $E^{3}$ such that for each point $p \in G$ and for each neighborhood $N$ of $p$ there is an open set $U$ such that $p \in U$ and each simple closed curve in $U-S$ can be shrunk to a point in $N-G$, then Property $(A, G, S)$ holds.

LEMMA 4. Suppose $M$ is a connected 2-manifold in $E^{3}, p$ is a point of $M$, and $V$ is a component of $E^{3}-M$. For each positive number $\varepsilon$ there is a disk $D$ in $M$ and a 2-sphere $S$ such that $p \in \operatorname{Int} D, D \subset S$, diam $S<\varepsilon$, and $D$ is on the boundary of $V \cap \operatorname{Int} S$. 
In this paper we consider connected 2-manifolds in $E^{3}$ and use the fact that each such manifold has exactly two complementary domains. If $S$ is a 2-sphere, we let Int $S$ be the bounded component of $E^{3}-S$, and we denote the unbounded component of $E^{3}-S$ by Ext $S$. A connected 2-manifold $M$ is tame if and only if $M$ is tame from each of its complementary domains [1], [3], [10], [18].

The set $Y$ is locally simply connected at a point $p$ of the closure of $Y$ if for each neighborhood $N$ of $p$ there is an open set $U$ containing $p$ such that each map of a simple closed curve into $U \cap Y$ is homotopic to a constant in $N \cap Y$. Using [4, Theorem 2.1] and [5, Theorem 1], Burgess has established that a connected 2-manifold is tame from a complementary domain $V$ if and only if $V$ is locally simply connected at each point of the manifold [11, Theorem 4]. Many of our proofs depend on this result.

If $D$ is a disk, we let Int $D=D-\operatorname{Bd} D$, where $\mathrm{Bd} D$ is the boundary of $D$. Rather than writing " $\varepsilon$ is a positive number" we sometimes write " $\varepsilon>0$ ". The symbol $N(R, \varepsilon)$, where $R$ is a point set and $\varepsilon>0$, stands for the set of all points that are within a distance $\varepsilon$ of some point of $R$.

We use $\mathrm{Cl}(R)$ to denote the closure of the set $R$, and we indicate that the diameter of $R$ is less than $\varepsilon$ by writing "diam $R<\varepsilon$ ". When we say that $f$ is a map we mean that $f$ is a continuous function.

2. Local spanning around arcs. We say that a connected 2-manifold $M$ can be locally spanned in a complementary domain $V$ around arcs if and only if for each $\varepsilon>0$ and for each arc $A$ in $M$ there is a disk $R$ in $M$ such that $A \subset \operatorname{Int} R$, $R \subset N(A, \varepsilon)$, and for each positive number $\alpha$ there exists a disk $D$ in $V$ so that $D \subset N(A, \varepsilon)$ and $\mathrm{Bd} R$ can be shrunk to a point in $N(\mathrm{Bd} R, \alpha)+D$. If $U$ is an open set in $M$, we say $U$ can be locally spanned in $V$ around arcs if the above conditions hold for each $\operatorname{arc} A$ in $U$.

THEOREM 1. If $V$ is a complementary domain of a 2-sphere $S$ in $E^{3}, U$ is an open subset of $S$, and $U$ can be locally spanned in $V$ around arcs, then $V$ is locally simply connected at each point of $U$.

Proof. Let $p$ be a point of $U$, let $N$ be a ncighborhood of $p$ such that $S-N \neq \varnothing$, and let $K$ be a disk in $U$ such that $p \in \operatorname{Int} K \subset K \subset N$. Let $O_{1}$ be an open set in $N$ such that $p \in O_{1}$ and $O_{1} \cap S$ lies in Int $K$. It follows from Theorem 4.1 of [9] that we can find an open set $O_{2}$ in $O_{1}$ such that $p \in O_{2}$ and for each map of Bd $K$ into $O_{2} \cap V$ there is a Cantor set $C$ on $S$ so that the map can be extended to map $K$ into $O_{1} \cap V+C \cap O_{1}$. Let $f$ be a map of $\mathrm{Bd} K$ into $O_{2} \cap V$ and extend $f$ to map $K$ into $O_{1} \cap V+C \cap O_{1}$, where $C$ is some Cantor set on $S$. We also use $f$ to denote the extension of $f$.

There is an $\operatorname{arc} A$ in Int $K$ such that $A$ contains $O_{1} \cap C$ [19]. Let $X$ be an arc from a point $b$ in $f(\operatorname{Bd} K)$ to a point $q$ in $S-N$ so that $X-q \subset V$. Choose a positive number $\varepsilon$ subject to the the following restrictions: 
(1) $\varepsilon<\rho(K, X)$.

(2) $\varepsilon<\rho(\mathrm{Bd} N, K)$.

(3) $\varepsilon<\rho(f(\mathrm{Bd} K), S)$.

Using the hypothesis, we let $R$ be a disk on $S$ such that $A \subset \operatorname{lnt} R, R \subset N(A, \varepsilon)$, and for each positive number $\alpha$ there is a disk $D$ in $V$ such that $D \subset N(A, \varepsilon)$ and $\mathrm{Bd} R$ can be shrunk to a point in $N(\mathrm{Bd} R, \alpha)+D$. Let $c$ be a point of $A$, and let $Z$ e an arc from $c$ to $q$ so that

$$
Z-(c+q) \subset E^{3}-(S+V) .
$$

Choose a positive number $\alpha$ such that

$$
\alpha<\rho(\operatorname{Bd} R, f(K)+Z+X+A) .
$$

Let $D$ be a disk in $V$ such that $D \subset N(A, \varepsilon)$ and $B d R$ can be shrunk to a point in $N(\operatorname{Bd} R, \alpha)+D$. Since $D \subset N(A, \varepsilon)$, it follows from (3) that $f(\operatorname{Bd} K) \cap D=\varnothing$. This permits us to use Lemma 2 to obtain a map $g$ of $K$ into $E^{3}$ such that $g|\mathrm{Bd} K=f| \mathrm{Bd} K, g(K) \subset f(K)+$ Int $D$, and $g(K)-D$ is connected.

It is our contention that $g(K) \subset N \cap V$. First we show $g(K) \subset N$. From $A \subset K$, $D \subset N(A, \varepsilon)$, and (2), it follows that $D \subset N$. Since $f(K) \subset O_{1}$, we know $f(K) \subset N$. Then $g(K) \subset N$ follows from $g(K) \subset f(K)+$ Int $D$.

Now we show that $g(K) \subset V$. Since $g(K)$ is connected and intersects $V, g(K)$ will lie in $V$ provided $g(K)$ does not intersect $S$. Suppose $g(K)$ does intersect $S$. Since $D \subset V$ it follows that the connected set $g(K)-D$ intersects $S$. There is an arc $Y$ from $b$ to a point $d$ in $A$ such that $Y \subset g(K)-D$ and $Y-d \subset V$. Let $W$ be the arc in $A$ from $c$ to $d$. In $X+Y+Z+W$ there is a simple closed curve $L$ which contains $W+Z$ and a subarc of $Y$ which contains $d$. Then $L$ links Bd $R$ [7, Theorem 3.3], so $\mathrm{Bd} R$ cannot be shrunk to a point in the complement of $L$ by Lemma 1 . This means that $L$ must intersect either $D$ or $N(\operatorname{Bd} R, \alpha)$. It follows from (1) and (4) that $D$ does not intersect $X+Z$. Since $W \subset S, D$ does not intersect $W$; and $Y \subset g(K)-D$. Then $D$ does not intersect $L$. It follows from (5) that $L \cap N(\operatorname{Bd} R, \alpha)=\varnothing$, so we have a contradiction. Hence $g(K) \subset V$.

Thus $g(K) \subset N \cap V$, so that $V$ is locally simply connected at $p$. It follows that $V$ is locally simply connected at each point of $U$.

THEOREM 2. If $M$ is a connected 2-manifold in $E^{3}, V$ is a component of $E^{3}-M$, and $M$ can be locally spanned in $V$ around arcs, then $M$ is tame from $V$.

Proof. Let $p \in M$. From Lemma 4 we obtain a disk $K$ in $M$ and a 2-sphere $S$ such that $p \in \operatorname{Int} K \subset S$ and $K$ is on the boundary of $V \cap \operatorname{Int} S$. We will show that Int $K$ can be locally spanned in Int $S$ around arcs. For this purpose we let $A$ be an arc in Int $K$ and let $\varepsilon>0$. We assume that $\varepsilon<\rho(A, S-K)$. From the hypothesis it follows that there is a disk $R$ in $M$ such that $A \subset \operatorname{Int} R, R \subset N(A, \varepsilon)$, and for each $\alpha>0$ there is a disk $D$ in $V$ so that $D \subset N(A, \varepsilon)$ and $\mathrm{Bd} R$ can be 
shrunk to a point in $N(\mathrm{Bd} R, \alpha)+D$. From our choice of $\varepsilon, R \subset \operatorname{Int} K$. We will choose $\alpha>0$ so that if $D$ is chosen as above relative to this $\alpha$, then $D \subset \operatorname{Int} S$. This will show that Int $K$ can be locally spanned in Int $S$ around arcs, and it will follow from Theorem 1 that Int $S$ is locally simply connected at points of Int $K$. Then $V$ will be locally simply connected at $p$, and Theorem 2 will follow from Theorem 4 of [11].

Since $K \subset \operatorname{Bd}(V \cap \operatorname{Int} S)$, we may choose $\alpha$ so $\mathrm{Cl}(N(\operatorname{Bd} R, \alpha)) \cap V \subset \operatorname{Int} S$. We also choose $\alpha$ so $\operatorname{Bd} R$ cannot be shrunk to a point in $N(\operatorname{Bd} R, \alpha)$, so that $D \cap \mathrm{Cl}((\operatorname{Bd} R, \alpha)) \neq \varnothing$. Then $D \cap \operatorname{Int} S \neq \varnothing$. Since $D \subset N(A, \varepsilon)$ and $D \subset V$, $D \cap S=\varnothing$. Then $D \subset \operatorname{Int} S$.

3. Local spanning on tame simple closed curves. Let $p$ be a point of a connected 2-manifold $M$ and let $V$ be a component of $E^{3}-M$. Then $M$ can be locally spanned at $p$ in $V$ on tame simple closed curves if and only if for each positive number $\varepsilon$ there is an $\varepsilon$-disk $R$ on $M$ such that $p \in \operatorname{Int} R, \operatorname{Bd} R$ is tame, and for each positive number $\alpha$ there is an $\varepsilon$-disk $D$ in $V$ so that $\mathrm{Bd} R$ can be shrunk to a point in $N(\operatorname{Bd} R, \alpha)+D$. If $U$ is a subset of $M$, we say that $U$ can be locally spanned in $V$ on tame simple closed curves if the conditions of the above definition are satisfied for each $p \in U$.

THEOREM 3. If $V$ is a complementary domain of a 2-sphere $S$ in $E^{3}$ and $U$ is an open subset of $S$ such that $U$ can be locally spanned in $V$ on tame simple closed curves, then $V$ is locally simply connected at each point of $U$.

Proof. Let $p \in U$, and let $N$ be a neighborhood of $p$ so that $S-N \neq \varnothing$. Let $K$ be a disk in $U$ such that $p \in \operatorname{Int} K$ and $K \subset N$. Let $O_{1}$ be an open set such that $O_{1} \cap S \subset$ Int $K, p \in O_{1}$, and $O_{1} \subset N$.

We apply Theorem 11 of [17] to obtain an open set $O_{2}$ such that if $f$ is a map of Bd $K$ into $O_{2} \cap V$ and if $F$ is a closed subset of $S$ satisfying Property $(*, F, S)$, then $f$ can be extended to map $K$ into $O_{1}-F$ so that $f(K) \cap S \subset \boldsymbol{\Sigma} H_{i}$, where $H_{1}, H_{2}, \cdots, H_{t}$ is a finite collection of disjoint disks in $\left(O_{1} \cap S\right)-F$.

Let $f$ be a map of Bd $K$ into $O_{2} \cap V$. For convenience we assume $V=$ Ext $S$. There is an $\operatorname{arc} X$ from a point $b$ in $f(\mathrm{Bd} K)$ to a point $q$ in $S-N$ so that $X-q \subset$ Ext $S$. We choose a positive number $\varepsilon$ so that

(1) $3 \varepsilon<\rho(K, X)$,

(2) $3 \varepsilon<\rho(\mathrm{Bd} N, K)$, and

(3) $2 \varepsilon<\rho(f(\operatorname{Bd} K), S)$.

For each point $r \in K$, we let $R_{r}$ be an $\varepsilon$-disk on $S$ so that $r \in \operatorname{Int} R_{r}, \mathrm{Bd} R_{r}$ is tame, and for each $\alpha>0$ there is an $\varepsilon$-disk $D_{r}$ in Ext $S$ such that Bd $R_{r}$ can be shrunk to a point in $N\left(\mathrm{Bd} R_{r}, \alpha\right)+D_{r}$. Since $K$ is compact and $\left\{\right.$ Int $\left.R_{r}\right\}$ is an open cover for $K$, there is a finite subcollection $R_{1}, R_{2}, \cdots, R_{n}$ of the $R_{r}^{\prime}$ s so that $K \subset \Sigma$ Int $R_{i}$.

Corollary 1 of [12] shows that Property (*, Bd $R_{i}$ ) is satisfied for each $i$, 
since $\mathrm{Bd} R_{i}$ is a tame finite graph. Let $F=\Sigma \mathrm{Bd} R_{i}$. It follows from Theorem 17 of [17] (or from Theorem 8.3 of [9]) that Property $(*, F)$ is satisfied. Then from Theorem 16 of [17] we have Property $(*, F, S)$ satisfied.

Using the definition of $\mathrm{O}_{2}$, we extend $f$ to map $K$ into $O_{1}-F$ such that $f(K) \cap S \subset \Sigma H_{i}$, where $H_{1}, H_{2}, \cdots, H_{t}$ is a finite collection of disjoint disks in $\left(\left(O_{1} \cap S\right)-F\right)$. Now we apply Lemma 2 to obtain a map $g_{0}$ of $K$ into $E^{3}$ such that $g_{0} \mid \mathrm{Bd} K=f, g_{0}(K) \subset f(K)+\Sigma$ Int $H_{i}$, and $g_{0}(K)-\Sigma H_{i}$ is connected. The following conditions are easily verified:

(4) $g_{0} \mid \mathrm{Bd} K=f$.

(5) $g_{0}(K) \cap F=\varnothing$.

(6) $g_{0}(K) \cap S \subset \Sigma H_{i} \subset O_{1} \cap S \subset \operatorname{Int} K$.

(7) $g_{0}(K) \subset O_{1} \cap(K+$ Ext $S) \subset N \cap(K+$ Ext $S)$.

We will make $n$ successive adjustments on the map $g_{0}$ to obtain a map $g_{n}$ of $K$ into $N \cap$ Ext $S$ such that $g_{n} \mid$ Bd $K=f$. For each $i$, let $R_{i}^{\prime}$ be a disk in Int $R_{i}$ so that $R_{i} \cap g_{0}(K)$ lies in $R_{i}^{\prime}$. Condition (5) allows us to do this. Let $c_{i}$ be a point in $R_{i}^{\prime}$, and let $Z_{i}$ be an arc from $c_{i}$ to $q$ so that $Z_{i}-\left(c_{i}+q\right) \subset$ Int $S$.

Then $R_{i}^{\prime}, c_{i}$, and $Z_{i}$ are defined for each $i$ such that $1 \leqq i \leqq n$.

Let $\alpha_{1}$ be a positive number such that

(8) $\alpha_{1}<\rho\left(\operatorname{Bd} R_{1}, g_{0}(K)+Z_{1}+X+R_{1}^{\prime}\right)$ and

(9) $\alpha_{1}<\varepsilon$.

Let $D_{1}$ be an $\varepsilon$-disk in Ext $S$ so that $\mathrm{Bd} R_{1}$ can be shrunk to a point in $N\left(\operatorname{Bd} R_{1}, \alpha_{1}\right)+D_{1}$. From (9), (4), and (3) it follows that $g_{0}(\operatorname{Bd} K) \subset E^{3}-D_{1}$; hence we can apply Lemma 2 to obtain a new map $g_{1}$ of $K$ into $E^{3}$ so that $g_{1}\left|\mathrm{Bd} K=g_{0}\right| \mathrm{Bd} K, g_{1}(K) \subset g_{0}(K)+$ Int $D_{1}$, and $g_{1}(K)-D_{1}$ is connected. Since $g_{0}(K)$ lies in $K+$ Ext $S$ it follows that $g_{1}(K) \subset K+$ Ext $S$.

We continue this procedure inductively, defining a finite collection of positive numbers $\alpha_{1}, \alpha_{2}, \alpha_{3}, \cdots, \alpha_{n}$; a finite collection of disks $D_{1}, D_{2}, \cdots, D_{n}$; and a finite sequence of maps $g_{0}, g_{1}, \cdots, g_{n}$ such that $g_{i}$ takes $K$ into $K+$ Ext $S$ and the following conditions hold for each $i(1 \leqq i \leqq n)$ :

(10) $\alpha_{i}<\varepsilon$.

(11) $\alpha_{i}<\rho\left(\operatorname{Bd} R_{i}, g_{i-1}(K)+R_{i}^{\prime}+Z_{i}+X\right)$.

(12) $D_{i} \subset \operatorname{Ext} S$.

(13) $\operatorname{diam} D_{i}<\varepsilon$.

(14) Bd $R_{i}$ can be shrunk to a point in $N\left(\mathrm{Bd} R_{i}, \alpha_{i}\right)+D_{i}$.

(15) $g_{i}\left|\mathrm{Bd} K=g_{i-1}\right| \mathrm{Bd} K=f$.

(16) $g_{i}(K) \subset g_{i-1}(K)+\operatorname{Int} D_{i}$.

(17) $g_{i}(K)-D_{i}$ is connected.

It is our contention that for each $i(1 \leqq i \leqq n), g_{i}(K)$ does not intersect $R_{i}$. Suppose $g_{j}(K)$ intersects $R_{j}$, where $1 \leqq j \leqq n$. Then $g_{j}(K)-D_{j}$ must intersect $R_{j}^{\prime}$, from (12) and the definition of $R_{j}^{\prime}$. Since $g_{j}(K)-D_{j}$ is arcwise connected, we let $Y_{j}$ be an arc in $g_{j}(K)-D_{j}$ from the point $b$ in $g_{0}(\mathrm{Bd} K)$ to a point $d_{j}$ in $R_{j}^{\prime}$. Since $g_{j}(K)-D_{j} \subset S+\operatorname{Ext} S$, we may choose $Y_{j}$ so that 
(18) $Y_{j}-d_{j} \subset(S+$ Ext $S)-\left(R_{j}+D_{j}\right)$.

It follows from (16) and the fact that $Y_{j} \subset g_{j}(K)-D_{j}$ that

(19) $Y_{j} \subset g_{j-1}(K)$.

Let $\mathrm{W}_{j}$ be an arc in $R_{j}^{\prime}$ from $c_{j}$ to $d_{j}$. Now $X+W_{j}+Y_{j}+Z_{j}$ contains a simple closed curve $L$ which links Bd $R_{j}$ [7, Theorem 3.3]. From (11) and (19) there can be no point of $L$ in $N\left(\operatorname{Bd} R_{j}, \alpha_{j}\right)$. Obviously $Z_{j}+W_{j}+Y_{j}$ does not intersect $D_{j}$; and from (10), (14), (13), and (1) we see that $X$ cannot intersect $D_{j}$. Then $L$ does not intersect $D_{j}$. From (14), Bd $R_{j}$ can be shrunk to a point in the complement of $L$, but this contradicts Lemma 1 . Hence $g_{i}(K)$ does not intersect $R_{i}$ for each $i$ such that $1 \leqq i \leqq n$.

From (16) and (12) we know that $g_{i}(K) \cap S \subset g_{i-1}(K) \cap S$. Then it follows from the preceding paragraph that $g_{n}(K)$ does not intersect $\Sigma R_{i}$. From (6) and the fact that $K \subset \Sigma R_{i}$, we see that $g_{n}(K)$ does not intersect $S$. Since $g_{n}(\operatorname{Bd} K)$ lies in Ext $S$, it follows that $g_{n}(K) \subset$ Ext $S$.

Each point of $R_{i}$ is within $\varepsilon$ of $K$ so it follows from (10), (13) and (14) that each point of $D_{i}$ is within $3 \varepsilon$ of $K$. Then from (2) we have $D_{i} \subset N(1 \leqq i \leqq n)$. From (16) and (7) we see that $g_{n}(K) \subset N$. Since we have shown that $f=g_{n} \mid \operatorname{Bd} K$, $f$ can be shrunk to a point in $N \cap$ Ext $S$. This shows that Ext $S$ is locally simply connected at $p$ and consequently at each point of $U$.

THEOREM 4. If $M$ is a connected 2-manifold in $E^{3}$ and $V$ is a component of $E^{3}-M$ such that $M$ can be locally spanned in $V$ on tame simple closed curves, then $M$ is tame from $V$.

Proof. Let $p \in M$. From Lemma 4 we obtain a disk $K$ in $M$ and a 2-sphere $S$ such that $p \in \operatorname{Int} K \subset S$ and $K$ is on the boundary of $V \cap$ Int $S$. We will show that Int $K$ can be locally spanned in Int $S$ on tame simple closed curves. For this purpose we let $q \in$ Int $K$ and let $\varepsilon$ be a positive number. We assume that $2 \varepsilon<\rho(q, S-K)$. From the hypothesis it follows that there is an $\varepsilon$-disk $R$ in $M$ so that $q \in \operatorname{Int} R, \mathrm{Bd} R$ is tame, and for each $\alpha>0$ there is an $\varepsilon$-disk $D$ in $V$ so $\mathrm{Bd} R$ can be shrunk to a point in $N(\mathrm{Bd} R, \alpha)+D$. It follows from our choice of $\varepsilon$ that $R \subset$ Int $K$. We will choose a positive number $\alpha$ so that a disk $D$ chosen as above relative to this $\alpha$ will lie in Int $S$. As in the proof of Theorem 2, we restrict $\alpha$ so that $D$ must intersect Int $S$. We also require that $\alpha<\varepsilon$ so some point of $D$ is within $\varepsilon$ of $R$. Then $D$ is in $N(R, 2 \varepsilon)$; hence $D \subset$ Int $S$. Thus Int $K$ can be locally spanned in Int $S$ on tame simple closed curves.

It follows from Theorem 3 that Int $S$ is locally simply connected at each point $q \in$ Int $K$. Then $V$ is locally simply connected at each point $p \in M$, and we apply Theorem 4 of [11] to see that $M$ is tame from $V$.

THEOREM 5. If $M$ is a connected 2-manifold in $E^{3}, V$ is a component of $E^{3}-M$, and for each $p \varepsilon M$ and for each $\varepsilon>0$ there is an $\varepsilon$-disk $R$ in $M$ such that $p \in \operatorname{Int} R$, $\mathrm{Bd} R$ is tame, and for each positive number $\alpha$ there is an $\varepsilon$-diskD in V and a homeo- 
morphism of $\mathrm{Bd} R$ onto $\mathrm{Bd} D$ which moves no point as much as a distance $\alpha$, then $M$ is tame from $V$.

Proof. Theorem 5 is a corollary of Theorem 4 . To see this we observe that if there is a homeomorphism $h$ of the simple closed curve $\mathrm{Bd} R$ onto the simple closed curve $\mathrm{Bd} D$ such that $h$ moves no point as much as $\alpha$, then $\operatorname{Bd} R$ and $\operatorname{Bd} D$ are homotopic in $N(\mathrm{Bd} R, \alpha)$. Then $\mathrm{Bd} R$ can be shrunk to a point in $N(\mathrm{Bd} R, \alpha)+D$.

REMARK. We state Theorem 5 so that the relation between Theorem 4 and the question in $\S 1$ is more apparent. Theorem 4 falls short of answering that question only because of the hypothesis that $\mathrm{Bd} R$ is tame.

4. Local spanning missing Cantor sets. Let $p$ be a point of the connected 2-manifold $M$ in $E^{3}$ and let $V$ be a component of $E^{3}-M$. We say that $M$ can be locally spanned at $p$ in $V$ missing Cantor sets if and only if for each $\varepsilon>0$ and for each Cantor set $C$ in $M$ there is an $\varepsilon$-disk $R$ in $M$ such that $p \in \operatorname{Int} R$, $\operatorname{Bd} R \cap C=\varnothing$, and for each $\alpha>0$ there is an $\varepsilon$-disk $D$ in $V$ so that $\mathrm{Bd} R$ can be shrunk to a point in $N(\operatorname{Bd} R, \alpha)+D$. If $U$ is a subset of $M$ we say that $U$ can be locally spanned in $V$ missing Cantor sets if the conditions of the above definition are satisfied for each $p \in U$.

THEOREM 6. If $V$ is a complementary domain of a 2-sphere $S$ in $E^{3}$ and $U$ is an open subset of $S$ such that $U$ can be locally spanned in $V$ missing Cantor sets, then $V$ is locally simply connected at each point of $U$.

Proof. Let $p \in U$. We assume for convenience that $V=\operatorname{Ext} S$; then define $N$, $K, O_{1}, O_{2}, f$, and $C$ just as in the proof of Theorem 1 . We select points $b$ and $q$ and construct an arc $X$ as in the proof of Theorem 3. Now we choose a positive number $\varepsilon$ satisfying Conditions (1), (2), and (3) of the proof of Theorem 3.

For each point $r \in K$, we let $R_{r}$ be an $\varepsilon$-disk in $S$ so that $r \in \operatorname{Int} R_{r}$, $C \cap \mathrm{Bd} R_{r}=\varnothing$, and for each $\alpha>0$ there is a disk $D_{r}$ in $V$ so that $\mathrm{Bd} R_{r}$ can be shrunk to a point in $N\left(\mathrm{Bd} R_{r}, \alpha\right)+D_{r}$. Since $K$ is compact we let $R_{1}, R_{2}, \cdots, R_{n}$ be a finite set of such $\varepsilon$-disks so that $K \subset \boldsymbol{\Sigma} R_{i}$.

If we let $F=\Sigma \mathrm{Bd} R_{i}$, we may follow the proof of Theorem 3, from the beginning of the paragraph which contains Condition (4) to the end of that proof, to complete the proof here.

THEOREM 7. If $M$ is a connected 2-manifold in $E^{3}$ and $V$ is a component of $E^{3}-M$ such that $M$ can be locally spanned in $V$ missing Cantor sets, then $M$ is tame from $V$.

Theorem 7 is established using Theorem 6 and the method given in the proofs of Theorems 2 and 4.

5. Sufficient conditions for closed sets to be tame. Let $G$ be a closed subset 
of a 2-sphere $S$ in $E^{3}$ such that the diameters of the components of $G$ have a positive lower bound.

THEOREM 8. If $G$ can be locally spanned in each complementary domain of $S$ on tame simple closed curves, then $G$ lies on a tame 2-sphere in $E^{3}$.

Proof. We will indicate how the proof of Theorem 3 can be modified to show that for each point $p \in G$ and each neighborhood $N$ of $p$ there is a neighborhood $U$ of $p$ so that each simple closed curve in $U-S$ can be shrunk to a point in $N-G$. It will then follow from Lemma 3 that Property $(A, G, S)$ is satisfied. Then we apply Theorems 10 and 6 of [17] to see that $G$ lies on a tame 2-sphere.

Let $p \in G$, and let $N$ be a neighborhood of $p$. Let $K$ be a disk on $S$ such that $p \in \operatorname{Int} K$ and $K \subset N$. Let $O_{1}$ be an open set such that $p \in O_{1}, O_{1} \cap S \subset \operatorname{Int} K$, and $O_{1} \subset N$. Now we apply Theorem 11 of [17] twice, once relative to Ext $S$ and once relative to Int $S$, to obtain an open set $\mathrm{O}_{2}$ containing $p\left(\mathrm{O}_{2}\right.$ is the intersection of the two open sets obtained from the two applications of Theorem 11 of [17]) such that if $f$ is a map of $\mathrm{Bd} K$ into $O_{2}-S$ and if $F$ is a closed subset of $S$ satisfying $(*, F, S)$, then $f$ can be extended to map $K$ into $O_{1}-F$.

Let $f$ be a map of $\mathrm{Bd} K$ into $\mathrm{O}_{2}-S$. Then we assume for convenience that $f(\operatorname{Bd} K) \subset \operatorname{Ext} S$. We choose $b, q, X$ and $\varepsilon$ as in the proof of Theorem 3 .

For each point $r \in G \cap K$, we choose an $\varepsilon$-disk $R_{r}$ on $S$ such that $r \in \operatorname{Int} R_{r}$, $\mathrm{Bd} R_{r}$ is tame, and for each $\alpha>0$ there is an $\varepsilon$-disk $D_{r}$ in Ext $S$ such that $\mathrm{Bd} R_{r}$ can be shrunk to a point in $N\left(\operatorname{Bd} R_{r}, \alpha\right)+D_{r}$. We choose a finite subcollection $R_{1}, R_{2}, \cdots, R_{n}$ of these $R_{r}$ 's so that $G \cap K \subset \sum$ Int $R_{i}$.

Now we follow the proof of Theorem 3, from the beginning of the fifth paragraph to the end, to obtain a proof here. Of course the final map $g_{n}$ of $K$ will not take $K$ into $N \cap$ Ext $S$, but $g_{n}(K)$ will lie in $N-G$.

THEOREM 9. If $G$ can be locally spanned in each component of $E^{3}-S$ missing Cantor sets, then $G$ lies on a tame 2-sphere in $E^{3}$.

REMARK. The proof of Theorem 9 is a modification o the proof of Theorem 6; hence we do not present a separate proof here.

Let $p$ be a point of a 2-sphere $S$ in $E^{3}$, and let $V$ be a component of $E^{3}-S$. If for each $\varepsilon>0$ there are disks $R$ and $D$ such that $p \in \operatorname{Int} R \subset S$, Int $D \subset V$, $\operatorname{Bd} D=\operatorname{Bd} R$, and diam $(D+R)<\varepsilon$, then $S$ can be locally spanned at $p$ from $V$. Burgess proved that $S$ is tame from $V$ if $S$ can be locally spanned at each point of $S$ from $V$ [11, Theorem 8]. His proof may be modified to show that under the hypothesis of the next theorem, Property $(A, G, S)$ is satisfied, so that it follows from Theorems 10 and 6 of [17] that $G$ lies on a tame sphere. The modification needed is similar to the modification of the proof of Theorem 3 which we indicated in the proof of Theorem 8 . 
THEOREM 10. If $S$ can be locally spanned at each point of $G$ from each complementary domain of $S$, then $G$ lies on a tame 2-sphere in $E^{3}$.

6. A small sphere characterization of tame surfaces. In this section we give a sufficient condition for a subcontinuum $G$ of a 2 -sphere in $E^{3}$ to lie on a tame 2-sphere in $E^{3}$. We rely on the result that $G$ lies on a tame 2-sphere if Property $\left(A^{\prime}, G, S\right)$ holds [17]. Although we state our theorems for subcontinua of 2spheres, the results are also valid if $G$ is a closed subset of a 2-sphere such that the diameters of the components of $G$ have a positive lower bound.

In the special case where $G$ is $S$, we obtain a sufficient condition, which is also necessary, for a 2-sphere to be tame (Theorem 12). Using the method illustrated in $\S 6$ of [11], one can also characterize tame 2-manifolds in a 3-manifold with this property.

THEOREM 11. If $S$ is a 2-sphere in $E^{3}$ and $G$ is a subcontinuum of $S$ such that or each point $p \in G$ and for each $\varepsilon>0$ there is a 2-sphere $S^{\prime}$ such that $p \in \operatorname{Int} S^{\prime}$, $\operatorname{diam} S^{\prime}<\varepsilon$, and $S \cap S^{\prime}$ is a continuum satisfying Property $\left(*, S \cap S^{\prime}, S\right)$, then $G$ lies on a tame 2-sphere in $E^{3}$.

Proof. Let $p \in G$ and let $N$ be a neighborhood of $p$ such that $S-N \neq \varnothing$. We will show the existence of an open set $U$ containing $p$ such that if $J$ is an unknotted simple closed curve in $U-S$, then $J$ bounds a tame disk in $N-G$. Since $G$ is compact it will follow, in a manner similar to the proof of Lemma 3, that Property $\left(A^{\prime}, G, S\right)$ is satisfied. Then Theorem 11 will follow from Theorems 13 and 6 in [17]. Let $K$ be a disk on $S$ such that $p \in \operatorname{Int} K \subset K \subset N$, and let $O$ be an open set containing $p$ such that $O \cap S \subset \operatorname{Int} K$ and $O \subset N$. Using $O$ in place of the " $N$ " of Theorem 12 in [17], we obtain from Theorem 12 in [17] an open set $U$ containing $p$ such that if $J$ is an unknotted simple closed curve in $U-S$ and $F$ is a closed subset of $S$ satisfying Property $(*, F, S)$, then $J$ bounds a tame disk in $O-F$. Let $J$ be an unknotted simple closed curve in $U-S$. For convenience we assume $J \subset \operatorname{Ext} S$.

Choose a positive number $\varepsilon$ such that

(1) $\varepsilon<\rho(\mathrm{Bd} N, K)$ and

(2) $\varepsilon<\rho(S, J)$.

For each $q \in G \cap K$, we let $S_{q}$ be a 2 -sphere such that $q \in \operatorname{Int} S_{q}$, $\operatorname{diam} S_{q}<\varepsilon$, and $S \cap S_{q}$ is a continuum satisfying Property $\left(*, S_{q} \cap S, S\right)$, and we let $R_{q}$ be the component of $S-S_{q}$ which contains $q$. Since the collection $\left\{R_{q}\right\}$ covers the compact set $K \cap G$, there is a finite subcollection $R_{1}, R_{2}, \cdots, R_{n}$ of the $R_{q}$ 's such that $K \cap G \subset \Sigma R_{i}$. We let $S_{1}, S_{2}, \cdots, S_{n}$ be the subcollection of the $S_{q}$ 's such that $R_{i}$ is defined relative to $S_{i}$. Let $F_{i}=S_{i} \cap S$, and let $F=\Sigma F_{i}$. Since Property $\left(*, F_{i}, S\right)$ is satisfied for each $i$, it follows from Theorem 21 of [17] that Property $(*, F, S)$ also holds. 
Using the restrictions on $U$ assured by Theorem 12 of [17], we let $H_{0}$ be a tame disk in $O-F$ such that $J=\operatorname{Bd} H_{0}$. Since $O \cap S \subset$ Int $K$ we know that $H_{0} \cap S \subset$ Int $K$. We will define a finite sequence of tame disks $H_{0}, H_{1}, \cdots, H_{n}$ so that $\operatorname{Bd} H_{n}=J$ and $H_{n} \subset N-G$. For notational convenience we define $S_{0}=R_{0}=H_{-1}=\varnothing$. The $H_{i}$ 's are defined inductively so that for each $i$,

(3) $\mathrm{Bd} H_{i}=J$,

(4) $H_{i} \subset N$,

(5) $H_{i} \cap S_{i}=\varnothing$,

(6) $H_{i} \cap S \subset H_{i-1} \cap S$, and

(7) $H_{i} \cap \Sigma_{m=0}^{i} R_{m}=\varnothing$.

Conditions (3), (4), (5), (6), and (7) are satisfied if $i=0$.

Now we assume that $H_{i}$ has been defined to satisfy Conditions (3) through (7) for $i \leqq j-1$, where $1 \leqq j \leqq n$. With no loss in generality we may assume that $H_{j-1}$ is locally polyhedral modulo $J$, that $S_{j}$ is locally polyhedral modulo $F_{j}$, and that $S_{j}-F_{j}$ and $H_{j-1}-J$ are in general position [2]. Since $H_{0} \subset 0-F$ and (6) is true for $i \leqq j-1$, it follows that $H_{j-1}$ does not intersect $F_{j}$. Also from (2), $S_{j}$ does not intersect $J$; hence $H_{j-1} \cap S_{j}$ consists of a finite collection of disjoint simple closed curves. Let $C_{j-1}$ be the component of $H_{j-1}-S$ such that $J \subset C_{j-1}$, and let $J_{1}, J_{2}, \cdots, J_{t}$ be the simple closed curves in $\operatorname{Cl}\left(C_{j-1}\right) \cap S_{j}$. No $J_{m}$ intersects $F_{j}$ and $F_{j}$ is connected, so $J_{m}$ bounds a disk $D_{m}$ on $S_{j}$ such that $D_{m} \cap F_{j}=D_{m} \cap S=\varnothing$.

From (2) we see that $C_{j-1} \subset \operatorname{Ext} S_{j}$. We can construct a disk $H_{j}$ by properly filling in the $t$ holes in $C_{j-1}$. Each of these $t$ holes is bounded by some $J_{i}$. We assume the $J_{i}$, s have been ordered so that if $i<j$, then $D_{i}$ does not contain $D_{j}$. First we add $D_{1}$ to $C_{j-1}$ and then we move $D_{1}$ slightly into Ext $S_{j}$. Next we add and move $D_{2}$. We continue this process as it is described in [5, p. 297] until we obtain a disk $H_{j}$ satisfying Conditions (3) through (7), where $i=j$.

We have inductively defined a sequence of disks $H_{0}, H_{1}, \cdots, H_{n}$ so that Conditions (3) through (7) are satisfied for each $i$. Since $H_{n} \subset N$, it remains to be shown that $H_{n} \cap G=\varnothing$. Suppose $H_{n}$ intersects $G$. From Condition (6) we know that $H_{n} \cap S \subset H_{0} \cap S \subset$ Int $K$. Then $H_{n}$ intersects $S$ in $\Sigma R_{i}$, since $K \cap G \subset \Sigma R_{i}$. But this is impossible from (7), so $H_{n} \subset N-G$.

We have Property $\left(A^{\prime}, G, S\right)$ satisfied, so Theorems 13 and 6 in [17] show that $G$ is tame if it is nondegenerate. If $G$ is degenerate, then $G$ obviously lies on a tame sphere.

THEOREM 12. If $S$ is a 2-sphere in $E^{3}$ such that for each point $p \in S$ and for each $\varepsilon>0$ there is a 2-sphere $S^{\prime}$ such that $p \in \operatorname{Int} S^{\prime}$, diam $S^{\prime}<\varepsilon$, and $S \cap S^{\prime}$ is a continuum satisfying Property $\left(*, S^{\prime} \cap S, S\right)$, then $S$ is tame.

Proof. We apply Theorem 11 where $G=S$ to see that $S$ is tame.

REMARK. The proof of Theorems 11 and 12 can be adjusted to give a sufficient condition for $S$ to be tame from a complementary domain $V$ of $S$. In particular 
the following proposition can be established. If $S$ is a 2-sphere in $E^{3}$ and $V$ is a complementary domain of $S$ such that for each $p \in S$ and for each $\varepsilon>0$ there is an $\varepsilon$-disk $D$ such that $D \cap p=\varnothing, \mathrm{Bd} D \subset E^{3}-V, D \cap S$ is a continuum satisfying $(*, D \cap S, S)$, and $p$ is on the boundary of an $\varepsilon$-component of $V-D$, then $S$ is tame from $V$.

COROLlaRY 13. A 2-sphere $S$ in $E^{3}$ is tame if for each $p \in S$ and for each $\varepsilon>0$ there is a 2-sphere $S^{\prime}$ such that $p \in \operatorname{Int} S^{\prime}, \operatorname{diam} S^{\prime}<\varepsilon$, and any one of the following three statements is satisfied:

1. $S \cap S^{\prime}$ is a continuum which lies in a tame Sierpinski curve in $S$.

2. $S \cap S^{\prime}$ is a 1-dimensional continuum such that $S$ is locally tame at each point of $S \cap S^{\prime}$.

3. $S \cap S^{\prime}$ is a continuum irreducible with respect to separating $S$ and $S$ is locally tame at $S \cap S^{\prime}$.

Proof. Statement 3 implies $2[16$, p. 98]. If 2 holds then there is an open set $N$ containing $S \cap S^{\prime}$ such that $S$ is locally tame at each point of $N$. Then there is a Sierpiński curve $K$ in $N$ such that $S \cap S^{\prime} \subset K$. Hence $K$ is tame, and 2 implies 1. It follows from Theorem 12, that 1 implies $S$ is tame, since a tame Sierpiński curve $K$ on a 2 -sphere satisfies Property $(*, K, S)$. (See Theorem 8.2 of [9] and Theorem 16 of [17], or see Theorems 16 and 19 of [17] together with [20].)

\section{REFERENCES}

1. R. H. Bing, Locally tame sets are tame, Ann. of Math. 59 (1954), 145-158.

2. - , Approximating surfaces with polyhedral ones, Ann. of Math. 65(1957), 456-483.

3. - An alternative proof that 3-manifolds can be triangulated, Ann. of Math. 69 (1959), 37-65.

4. - , Conditions under which a surface in $E^{3}$ is tame, Fund. Math. 47 (1959), 105-139.

5. - , A surface is tame if its complement is 1-ULC, Trans. Amer. Math. Soc. 101 (1961), 294-305.

6. — , Each disk in $E^{3}$ contains a tame arc, Amer. J. Math. 84(1962), 583-590.

7. — , Each disk in $E^{3}$ is pierced by a tame arc, Amer. J. Math. 84 (1962), 591-599.

8. — , Approximating surfaces from the side, Ann. of Math. 77 (1963), 145-192.

9. _- , Pushing a 2-sphere into its complement, Mich. Math. J. 11 (1964), 33-45.

10. Morton Brown, Locally fat imbeddings of topological manifolds, Ann. of Math. 75 (1962), 331-341.

11. C. E. Burgess, Characterizations of tame surfaces in E3, Trans. Amer. Math. Soc. 114 (1965), 80-97.

12. P. H. Doyle and J. G. Hocking, Some results on tame disks and spheres in E3, Proc. Amer. Math. Soc. 11 (1960), 832-836.

13. D. S. Gillman, Side approximation, missing an arc, Amer. J. Math. 85 (1963), 459-476.

14. O. G. Harrold, Jr., Locally peripherally unknotted surfaces in E3, Ann. of Math. 69 (1959), 276-290.

15. O. G. Harrold, Jr., H. C. Griffith, and E. E. Posey, A characterization of tame curves in 3-space, Trans. Amer. Math. Soc. 79 (1955), 12-35. 
16. Witold Hurewicz and Henry Wallman, Dimension theory, Princeton Univ. Press, Princeton, N. J., 1948.

17. L. D. Loveland, Tame subsets of spheres in $E^{3}$, Pacific J. Math. (to appear).

18. E. E. Moise, Affine structures in 3-manifolds. VIII, Invariance of knot-types; local tame

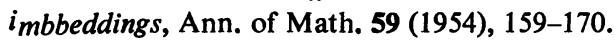

19. R. L. Moore and J. R. Kline, On the most general plane closed set through which it is possible to pass a simple continuous arc, Ann. of Math. 20 (1919), 218-223.

20. G. T. Whyburn, Topological characterization of the Sierpinski curve, Fund. Math. 45 (1958), 320-324.

The UNIVERSITY OF UTAH,

Salt Lake City, Utah

Utah STATE University,

I.OGAN. ITAAH 